\title{
Transmission Line Filters With Harmonic Parallel Foster Sections
}

\author{
J.A.G. Malherbe, P.D. Luus and J.C. Uys \\ Department of Electrical, Electronic and Computer Engineering \\ University of Pretoria, South Africa \\ jagm@up.ac.za
}

\begin{abstract}
Conventional planar wideband bandstop filters with rapid cutoff employ Cauer prototypes, but become non-realizable with Kuroda-Levy transforms for bandwidths of 150\% or more. Transmission line filters with harmonic stubs have been shown to be realizable at these bandwidths, but have limited performance ranges due to the fixed positions of the transmission zeros. In this paper, two structures that employ shunt Foster resonators that enable the shift of the transmission zeros, are described. Simulated and measured properties show that greatly improved performance can be realized while not adding to the complexity of the structure.
\end{abstract}

Key words: harmonic stub filter, bandstop filter, shunt Foster sections, pseudo-elliptic function response.

\section{Introduction}

Previously, microwave bandstop and bandpass filters were described that make use of open- or short circuited shunt stubs of which the lengths are in a harmonic ratio [1] [5]. In the 1:2:4 ratio filters, stub lengths are in an octave ratio of $l_{0}=\lambda_{0} / 4,2 l_{0}$ and $4 l_{0}$. The stubs contribute transmission zeros at frequencies of $\left\{f_{0}\right\}$ for $l_{0},\left\{0.5 f_{0}, 1.5 f_{0}\right\}$ for $2 l_{0}$ and $\left\{0.25 f_{0}, 0.75 f_{0}, 1.25 f_{0}, 1.75 f_{0}\right\}$ for $4 l_{0}$, giving 7 transmission zeros in all, before repeating the harmonic frequency response of the filter [3], [4].

The filters with shunt stubs with harmonic ratios of $1: 2: 3$ will similarly give transmission zeros at frequencies of $\left\{f_{0}\right\}$ for $l_{0},\left\{0.5 f_{0}, 1.5 f_{0}\right\}$ for $2 l_{0}$, and $\left\{0.333 f_{0}, f_{0}\right.$, $\left.1.667 f_{0}\right\}$ for $3 l_{0}$. Note that there are two zeros on either side of the centre frequency and a double zero at $f_{0}[5]$. The pole at $f_{0}$ contributed by the stub of length $4 l_{0}$ has now become a zero at $f_{0}$.

The occurrence of transmission zeros away from the centre frequency makes a pseudo-elliptic response possible. By adjustment of the stub impedances, and the impedance levels of the quarter-wave cascade lines separating them (unit elements, u.e.), superior passband match, rapid cutoff, and high stopband rejection can be obtained, almost equalling the performance of the Cauer filters; the latter are not physically realizable by means of Kuroda-Levy transforms for bandwidths on the order of $150 \%$ because of the occurrence of negative inductors [6].

Unfortunately, the application of the 1:2:4 and 1:2:3 structures are limited by the fact that the transmission zeros occur at fixed frequencies. This results in tradeoffs between passband return loss and stopband level.

In this paper, two new harmonic filters are described, that overcome some of the limitations of the filters with simple stubs, by making use of shunt Foster sections instead of simple stubs. Additional orders of freedom are introduced to the networks, and the performance is substantially improved. Examples of 1:2:4 and 1:2:3 stub ratio filters with shunt Foster sections are presented, including numerical modelling and measurements on constructed filters. It is shown that a substantial improvement in performance is achieved. 


\section{Shunt Foster Sections}

Fig. 1(a) shows a schematic of a simple single section shunt stub resonating at $0.5 f_{0}$, and length $2 l_{0}$. In Fig. 1(b) the single stub is replaced by shunt Foster section, a cascade of two unit elements, each of length $l_{0}$, and impedances $Z_{1}$ and $Z_{2}$, respectively.

The input impedance to the shunt Foster section is given by

$$
\begin{aligned}
Z_{\text {in }} & =Z_{1} \frac{\left(Z_{2} / S\right)+S Z_{1}}{Z_{1}+S\left(Z_{2} / S\right)}=\frac{1}{S} \frac{Z_{1} Z_{2}}{Z_{1}+Z_{2}}+S \frac{Z_{1}^{2}}{Z_{1}+Z_{2}} \\
& =Z_{C} / S+S Z_{L},
\end{aligned}
$$

where $S=j \tan \left(\frac{\pi}{2} \frac{f}{f_{0}}\right)$.

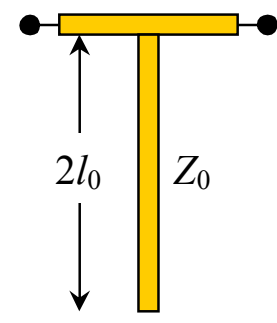

(a)

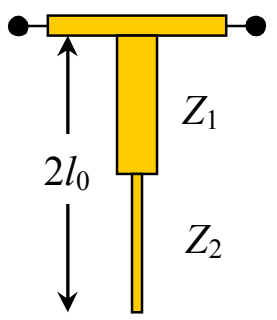

(b)

Figure 1 (a): Stub resonating at $f_{0} / 2$; (b): Shunt Foster section with Shifted transmission zeros.

The shunt Foster section resonates where the input impedance to the section is zero, or

$$
\begin{aligned}
0 & =Z_{C} / S_{00}+S_{00} Z_{L} \\
\tan \left(\frac{\pi}{2} \frac{f_{00}}{f_{0}}\right) & = \pm \sqrt{\frac{Z_{C}}{Z_{L}}}= \pm \sqrt{\frac{Z_{2}}{Z_{1}}} \\
f_{00} & =f_{0} \frac{2}{\pi} \tan ^{-1} \pm \sqrt{\frac{Z_{2}}{Z_{1}}} .
\end{aligned}
$$

If $Z_{2}=Z_{1}=Z_{0}$, then $f_{00}=f_{0} / 2$, as for the stub in Fig. 1(a).

$$
\text { For } \begin{aligned}
Z_{1} & >Z_{2} \\
f_{00} & =\left\{\left(f_{0} / 2-\delta\right),-\left(f_{0} / 2-\delta\right\}=\left\{\left(f_{0} / 2-\delta\right),-\left(f_{0} / 2-\delta\right)+2 \pi\right\}\right. \\
& =\left\{\left(f_{0} / 2-\delta\right),\left(3 f_{0} / 2+\delta\right\}\right.
\end{aligned}
$$

For $Z_{1}<Z_{2}$

$$
\begin{aligned}
f_{00} & =\left\{\left(f_{0} / 2+\delta\right),-\left(f_{0} / 2+\delta\right\}=\left\{\left(f_{0} / 2+\delta\right),-\left(f_{0} / 2+\delta\right)+2 \pi\right\}\right. \\
& =\left\{\left(f_{0} / 2+\delta\right),\left(3 f_{0} / 2-\delta\right)\right\}
\end{aligned}
$$


These two cases are illustrated graphically in Fig. 2. Without altering the lengths of the stubs, i.e. maintaining the symmetrical placement about the centre frequency, an additional order of freedom is introduced to adjust the ripple level by means of the positions of the transmission zeros. Note that the zeros can be shifted to either side of the initial resonance frequency.

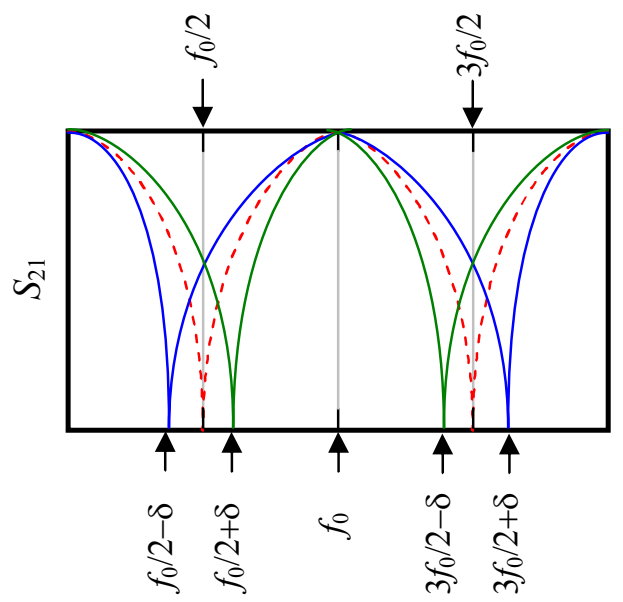

Figure 2 Transmission response of shunt Foster section, with transmission zeros shifted either side of $f_{0}$.

\section{Octave Ratio Filter (1:2:4)}

The octave ratio filter has three stubs in length ratio of 1:2:4 [4]. Fig. 3(a) shows a typical layout of an octave ratio filter with unmodified stubs, and the corresponding filter in which the stubs $Z_{2}$ and $Z_{3}$ have been replaced by Foster sections is shown in Fig. 3(b). Note that the stubs are spaced by two cascade unit elements, each of length $l_{0}$, as well as a feed u.e. of length $l_{0}$.

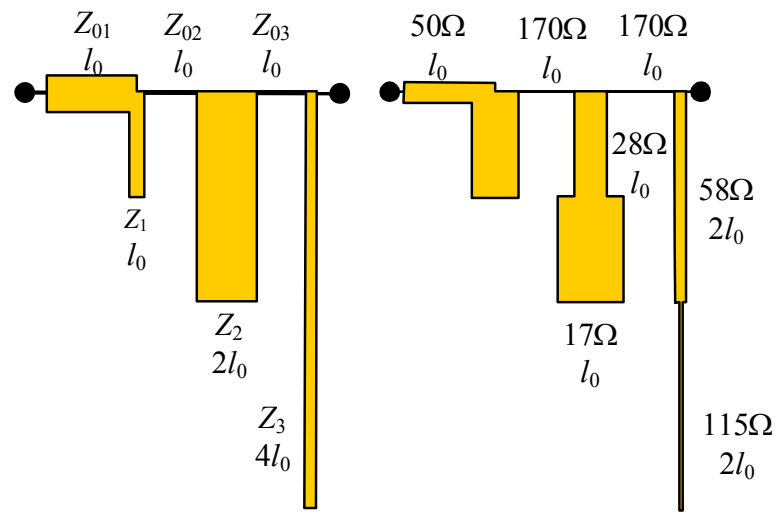

(a)

(b)

Figure 3 Structure of 1:2:4 filter without (a), and with Foster sections, (b).

A typical frequency response was calculated for a filter without Foster sections [4]. The trensmission zeros lie at $\left\{0.25 f_{0}, 0.5 f_{0}, 0.75 f_{0}, f_{0}, 1.25 f_{0}, 1.5 f_{0}\right.$, $\left.1.75 f_{0}\right\}$. As can be seen from Fig. 4, the outermost peaks in the stopband are high; the 
set of peaks between $0.5 f_{0}$ and $0.75 f_{0}$ and its mirror are depressed, and due to the single zero at the centre frequency, the centre peaks are agin high.

The stub $Z_{2}$ of length $2 l_{0}$ is modified into a shunt Foster section of two elements each $l_{0}$ long, and the stub $Z_{3}$ of length $4 l_{0}$ is split into two sections of length $2 l_{0}$. As an example of the type of frequency response that can be obtained, values of impedance $Z_{1}=28 \Omega$ and $Z_{2}=17 \Omega$, are chosen for the $2 l_{0} \mathrm{stub}$, as shown in Fig. 3(b). This causes the associated transmission zeros to shift from $\left\{0.5 f_{0}, 1.5 f_{0}\right\}$ to $\left\{0.421 f_{0}\right.$, $\left.1.579 f_{0}\right\}$; the first peak in the stopband is consequently reduced, and the second rises in level. Similarly, the stub of length $4 l_{0}$ is split into two sections of length $2 l_{0}$ and with characteristic impedances of $Z_{1}=58 \Omega$ and $Z_{2}=115 \Omega$. Bearing in mind that this section repeats its first half-wave response around $0.5 f_{0}$, and not $f_{0}$, the new resonant frequencies change to $\left\{0.303 f_{0}, 0.697 f_{0}, 1.303 f_{0}, 1.697 f_{0}\right\}$. The responses of this filter is compared to that of the filter wihout the Foster sections in Fig. 4.

Some of the zero shifts oppose each other, and a balance has to be struck between the rate of cutoff, the passband match, and the stopband attenuation level. Note that these values of $Z_{1}$ and $Z_{2}$ for the two Foster sections are not unique. There exists a very large range of impedances that will yield frequency responses of acceptable to excellent performance. This will be determined by the passband return loss and the stopband insertion loss, as well as the realizability of the elements. The rate of cutoff is also a variable but the various parameters are not independent.

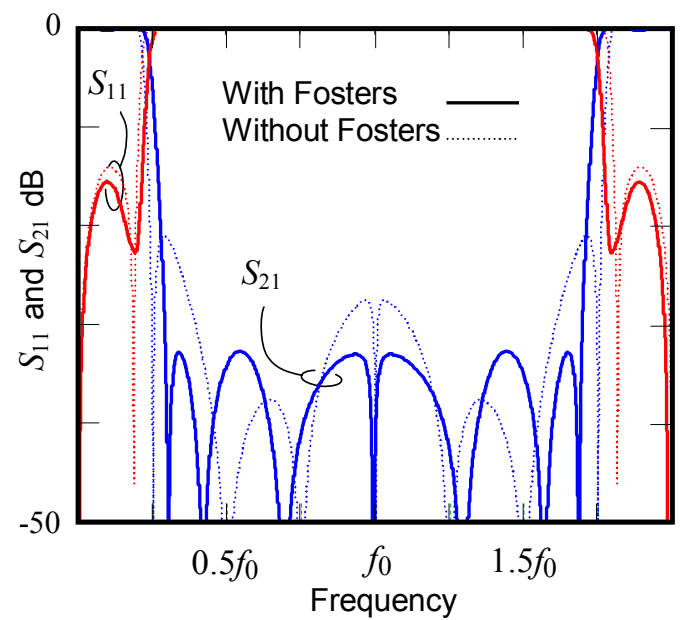

Figure 4 Comparison between the $S_{11}$ and $S_{21}$ responses of the 1:2:4 ratio stub length filters with and without shunt Foster sections.

\section{Fibonacci Ratio Filter (1:2:3)}

The 1:2:4-filter has only one transmission zero at the centre frequency, while a filter in which the element lengths are part of a Fibonacci seies with a 1:2:3 ratio has two transmission zeros at $f_{0}$. The structure of this filter is shown in Figs 5(a) and (b) for the filter without and with a Foster section, respectively.

The stub with length $3 l_{0}$ creates zeros at $0.333 f_{0}, f_{0}$ and $1.667 f_{0}$. Fig. 6 shows a typical response for a 1:2:3 ratio filter without a Foster section; in respect of the centre lobes, this is clearly superior to the 1:2:4 filter, although the rate of cutoff is not as rapid as in the latter case [5]. 
By replacing the $2 l_{0}$-stub with a shunt Foster section, a further degree of freedom is introduced. As an example, the transmission zeros at $\left\{0.5 f_{0}, 1.5 f_{0}\right\}$ were shifted to $\left\{0.544 f_{0}, 1.456 f_{0}\right\}$ by making $Z_{1}=19 \Omega$ and $Z_{2}=25 \Omega$, while the other impedances have been adjusted to obtain a desirable response with low passband loss and an equiripple stopband loss of $30 \mathrm{~dB}$. The transmission zeros at $0.333 f_{0}, f_{0}$ and $1.667 f_{0}$ have remained unchanged. The stub $Z_{3}$ cannot be split into a shunt Foster because it has a first half-wave resonance at $0.667 f_{0}$, and consequently the zero at $f_{0}$ would shift away, distorting the frequency response.

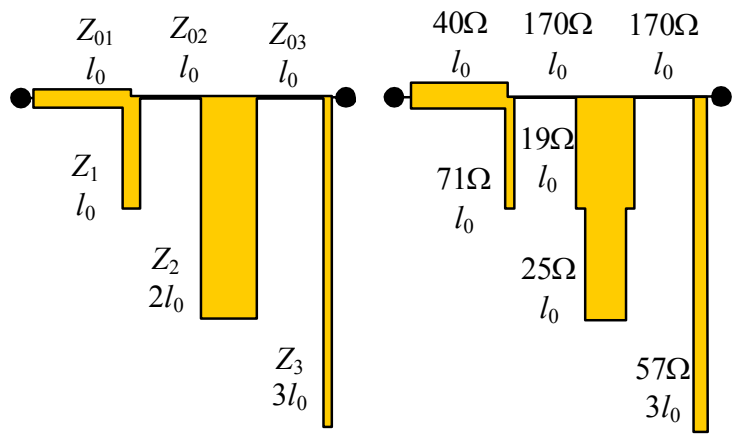

(a)

(b)

Figure 5 Structure of 1:2:3 stub ratio filter without (a), and with shunt Foster section, (b).

Fig. 6 compares the frequency response obtained using a 1-2-3 layout with one shunt Foster section to that of the network without a Foster. Note that once again there exists a vast number of impedance and zero position solutions that can affect the properties of the filter; these presented here are not necessarily the best solutions.

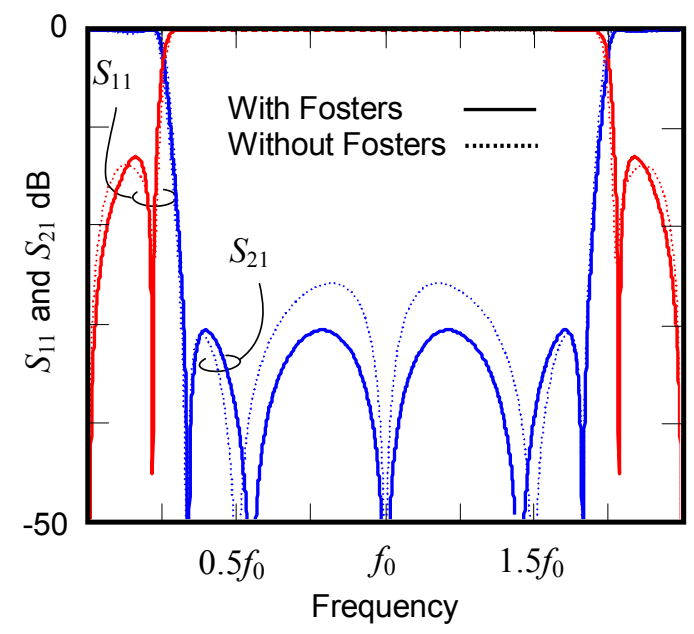

Figure 6 Comparison between the $S_{11}$ and $S_{21}$ responses of the 1:2:3 ratio stub length filters with and without shunt Foster sections.

\section{Numerical Performance Evaluation}

The performance of the resonant stub filters with Foster sections is evaluated against that of a Cauer filter with passband and stopband levels closest to the examples developed. In this instance, the Cauer filter response is used as a standard, even though it is known that Cauer filters become non-realizable at the bandwidths in 
question [6]. For both the filters illustrated, the nearest Cauer response is obtained and bandwidth scaled to coincide with that of the harmonic filter at the $-3 \mathrm{~dB}$ points. Because of the rapid rate of cutoff, this corresponds roughly to the pssband equiripple points.

The Cauer filter that most closely resembles the 1:2:4 filter in passband and stopband performance is designated $\mathrm{C} 0520, \Theta=55^{\circ}$ [7]; it has a passband reflection coefficient of $a_{\max }=-13.98 \mathrm{~dB}$, and stopband level of $a_{\min }=-32.2 \mathrm{~dB}$. Comparitive responses between the 1:2:4 filter and the Cauer filter is shown in Fig. 7.

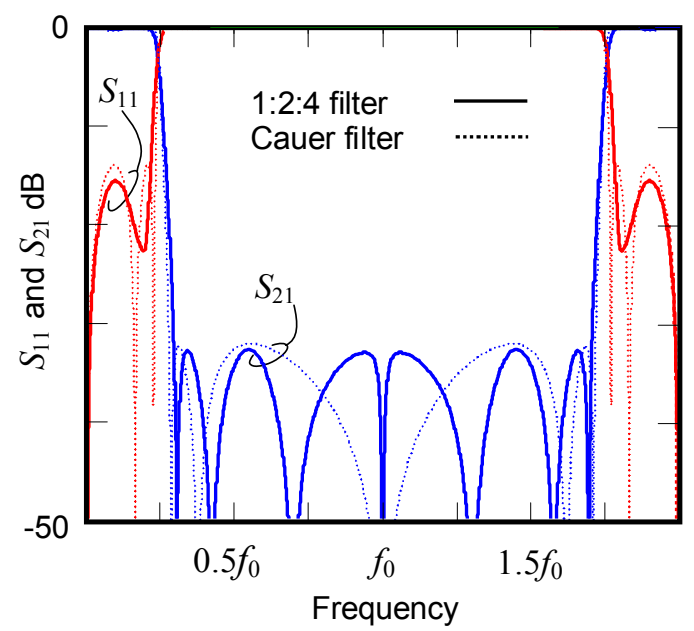

Figure 7 Comparison of 1:2:4 harmonic filter and Cauer filter response

The harmonic filter compares extremely well with the Cauer filter. The low frequency passband of the harmonic filter is slightly narrower than the Cauer prototype. The rate of cutoff is, however, just as rapid; passband bandwidth can be offset against the rate of cutoff by adjusting the impedances.

For the harmonic filter with 1:2:3 ratio stubs, a Cauer filter designated C0520, $\Theta=$ $57^{\circ}$ was chosen; it has a passband reflection coefficient of $a_{\max }=-13.98 \mathrm{~dB}$, and stopband attenuation level of $a_{\min }=-30.3 \mathrm{~dB}$. Responses are compared in Fig. 8 .

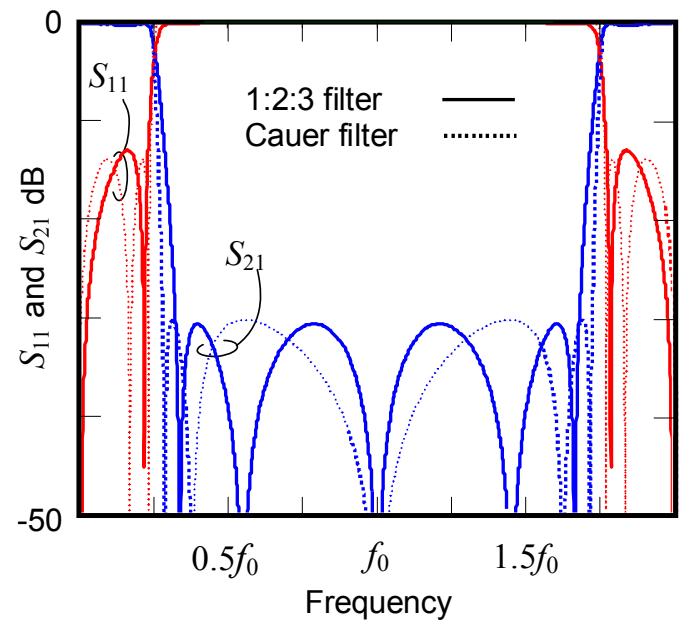

Figure 8 Comparison of 1:2:3 harmonic filter and Cauer filter response

The low frequency passband of the 1:2:3 filter is the same as that of the Cauer filter. However, it does not cut off as rapidly. Once again, passband bandwidth can be offset against the rate of cutoff by adjusting the impedances. 


\section{Measured and Simulated Filter Performance}

Both the filters described in Figures 4 and 6 above were first simulated in Sonnet [8] and then constructed as microstrip circuits on RT Duroid 5880, with $\varepsilon_{\mathrm{r}}=2.2$, and dielectric thickness of $1.57 \mathrm{~mm}$.

The measured responses of $S_{11}$ and $S_{21}$ of the 1:2:4 filter are shown in Fig. 9. Also shown are the simulated responses as well as the calculated performance. Similar responses are given for the 1:2:3 filter in Fig. 10.

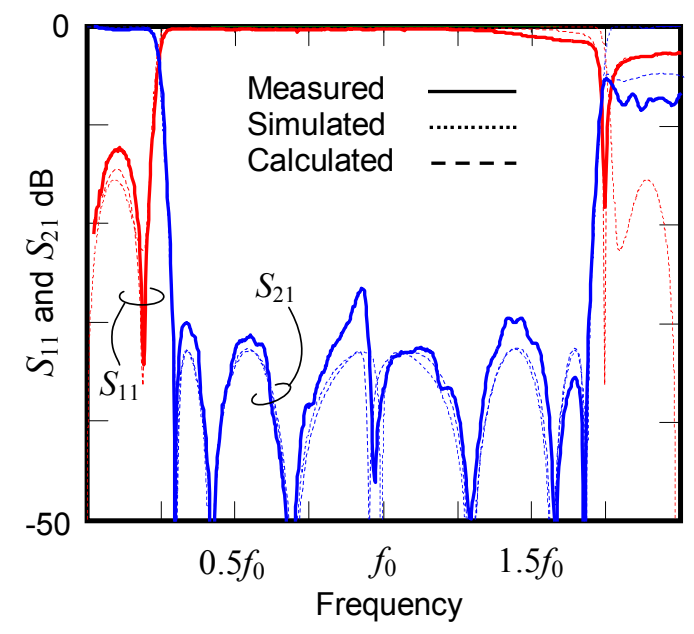

Figure 9 Measured $S_{11}$ and $S_{21}$ responses for the 1:2:4 filter.

This is compared to the calculated and the simulated responses (Sonnet).

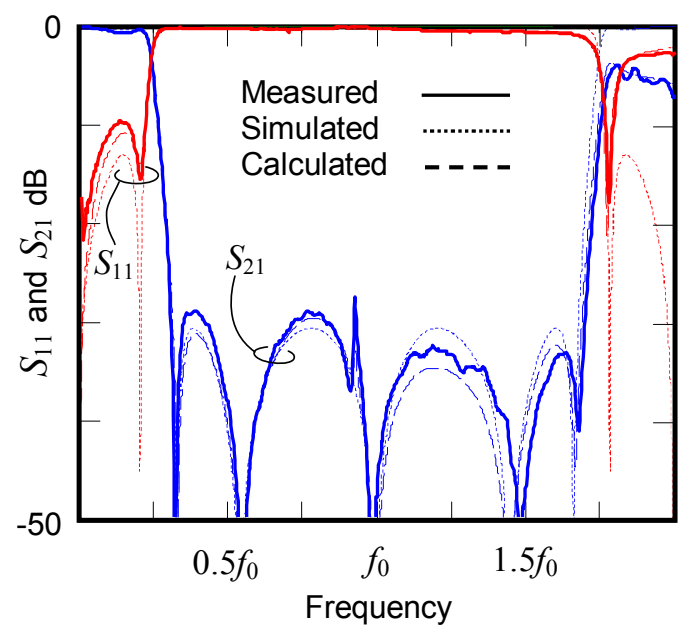

Figure 10 Measured $S_{11}$ and $S_{21}$ responses for the 1:2:3 filter. This is compared to the calculated and the simulated responses (Sonnet).

\section{Conclusion}

For both the simulated and measured responses, the preformance deteriorates at the high frequency end of the filter; as this is to be applied as a pseudo-lowpass filter, it is not of any concern. It is caused by the increased loss in the substrate material, as well 
as incresed dispersion from the microsrtip lines, especially in view of the extremities of impedance in the structure.

Tradeoffs exist between lower passband return loss, lower passband bandwidth, stopband attenuation level, and rate of cutoff. Of the multitude of possible solutions, two have been presented, that could be considered to be typical.

A comparison with the Cauer filter prototypes show responses that perform extremely well at bandwidths of the order of $150 \%$, where very few alternative planar designs with similar performance are available.

\section{References}

1. J.A.G. Malherbe and C. Reid, Double Resonant Stub Bandstop Filter with Pseudo-Elliptic Response, Electron Lett., 46 (2010), 508-509.

2. J.A.G. Malherbe, Pseudo-Elliptic Bandpass Filter with Sub-Harmonic Stubs, Electron Lett., 47 (2011), $196-198$.

3. J.A.G. Malherbe, Wideband Bandstop filter with Sub-Harmonic Stubs, Electron Lett., 47 (2011), $604-605$.

4. J.A.G. Malherbe, Wide band bandstop filter with harmonic stubs, in XIII Internat Symp Microwave Opt Technol. (ISMOT-2011), June 20 - 23, 2011.

5. J.A.G. Malherbe, Pseudo-elliptic bandstop filter with 1:2:3 harmonic ratio stubs, Electron Lett., to be published.

6. B.M. Schiffman and L. Young, Design Tables for an Elliptic-Function Band Stop Filter $(N=5)$, IEEE Trans Microwave Theory Tech, 14 (1966), 474-482.

7. R. Saal, Handbook of filter design, Berlin, Germany, AEG-Telefunken, 1979.

8. Sonnet ${ }^{\circledR}$, Sonnet Software Inc. 